\title{
ABSOLUTE MAGNITUDES OF RR LYRAE STARS
}

\author{
ROBERT F. CHRISTY \\ Physics Department, California Institute of Technology, Pasadena, Calif., U.S.A.
}

\section{Introduction}

In discussing the absolute magnitudes of RR Lyrae stars, I concur in the importance of the question but I would, at the outset, like to insert a word of caution: It seems to me most likely that the $M_{b}$ of RR Lyrae stars is not an immutable constant but depends on the original composition of the star. I will come back to this point later.

The principal point of view which I shall emphasize is rather different from that of most observers. The observer is usually interested in using the RR Lyrae stars as a means to calibrate distances and thereby looks out from the star to the Galaxy and then to the Cosmos. In contrast, the interests of the theorist look inward and he views the RR Lyrae stars as convenient fixed points for the comparison of theory with observation.

At present, the theory of stellar structure is tied to fitting the Sun. We all know the difficulties in the solar neutrino experiment which casts some doubt on whether we even understand the Sun. But we have few if any cases of evolved stars where we know $M, L, R$, to check our stellar evolution calculations.

We are now in desperate need of new fixed points where we can compare observation and the theory of stellar structure and evolution. The RR Lyrae or Cepheid type variables provide excellent examples for this comparison of observation and calculation. They are in late stages of evolution where tests are needed, they are readily identifiable, and well observed, and already the knowledge of the period provides a very precise mass-radius relationship so that a complete determination of the model will be provided by only a few additional measures.

\section{Relations to the Theory of Variable Stars}

The period of a Cepheid-type variable provides a very precise measure of the structure which can be employed to help interpret observations of variables. The most precise expression I have found is

$$
Q=0.0334+0.00034 \frac{\left(R / R_{\odot}\right)^{1.18}}{M / M_{\odot}},
$$

but the relation that is most useful is somewhat less accurate:

$$
P_{F}(\text { days })=0.021 \frac{\left(R / R_{\odot}\right)^{1.76}}{\left(M / M_{\odot}\right)^{0.72}}
$$

If we now introduce the relation between $M_{b}, R$, and $T_{e}$, we can eliminate $\left(R / R_{\odot}\right)$ 
and get

$$
-M_{b}=2.84 \log P_{F}+10 \log T_{e}+2.05 \log M / M_{\odot}-37.58 \text {. }
$$

It is important to remember that this relation uses theory only in the expression for the period which is very accurate and should lead to errors in $M_{b}$ less that 0.03 for periods less than 40 days. It is apparent that the principal uncertainty in applying this relation arises in determining $\log T_{e}$. Where necessary, I have used the relation $\log T_{e}=3.886-0.175\langle\mathrm{~B}-\mathrm{V}\rangle$, but I will largely express things in terms of $\log T_{e}$ in order to avoid introducing unnecessary approximate relations.

It is apparent from this relation that if we know $\log T_{e}$ and Mass (and of course $\log P$ ) we can deduce $M_{b}$. Alternatively we can examine a series of variables where relative $M_{b}, \log T_{e}$, and $\log P$ are known (such as in a globular cluster or the Magellanic Clouds) and determine relative values of Mass. This could tell us, for example whether Mass is constant or varying across the RR Lyrae gap. Clearly, the determination of mean $\log T_{e}$ from mean $\langle\mathrm{B}-\mathrm{V}\rangle$ and other colours is vital to this and most other attempts to relate variables to theory. Here more basic work by astronomers and stellar atmosphere theorists is needed to enable this determination to be made with appropriate allowance for the dependence on gravity and on metal content. Although much work has gone into this study, much more is needed. If we wish to determine $M_{b}$ to within 0.1 , we need $\log T_{e}$ to 0.01 .

If we now assume that we have the basic knowledge to determine $\log T_{e}$ accurately, we see that we need to know Mass or Radius if we are to determine $M_{b}$.

A very basic method of determining Radius is by some modification of the Wesselink method. By this, I mean to use information on changes in colour and luminosity to deduce the fractional change in radius. By then comparing with the absolute change in radius during this same time, obtained by integrating the velocity curve, we deduce the actual radius. This procedure is probably best applied during falling light when the atmosphere is most like a normal stellar atmosphere though of abnormally low $\mathrm{g}$. This method, however, entails some systematic errors in that it incorrectly assumes that the change in radius of the photosphere is the same as the distance moved by matter in the reversing layer. Actually the photosphere moves by considerably less than the material motions in the reversing layer would imply because it descends to greater depth at maximum radius, particularly for longer period Cepheids. This means that the method will consistently overestimate the radius. I have only estimated the correction for a 10 day Cepheid and guess that the Wesselink radius may be $10 \%$ in excess of the true mean radius. It remains an important problem for theorists who make dynamic models to evaluate the best way of using the Wesselink method and how to so correct the results to get the correct mean radius.

Recently I have found that the radius may be determined from non-linear pulsation calculations for certain variables which show bumps in the light or velocity curve that are characteristic of the Hertzsprung progression. So far, unfortunately, the method is much better in calculating velocity curves than light curves so that only a very few stars are suitable for application. The method gives $R / R_{\odot}=4.05 \times$ delay (days) and, 
where applicable, leads to $R$, and, coupled again with $T_{e}$, we get $M_{b}$. For example, for $S$ Nor I get $M_{b}=-3.80$, whereas Kraft from cluster fitting gets -4.05 . This lower $L$ corresponds to a radius smaller by 0.05 in the logarithm or $12 \%$ and a mass smaller by $40 \%$ than normal. An alternative implication is that the model calculations are wrong, and I estimate that this discrepancy would be eliminated by an increase of opacities by a factor of two in the range $50000 \mathrm{~K}$ to $500000 \mathrm{~K}$.

Finally, I would like to comment on another relationship involving the luminosity found in the nonlinear calculations. It has appeared that the lowest period $P_{\operatorname{Tr}}$ at which the fundamental will vibrate unstably, before the overtone takes over, is correlated with the luminosity,

$$
M_{\mathrm{bol}}=-0.52-4.46 \log P_{\mathrm{Tr}} .
$$

Applied to a few cases of globular clusters, this gave:

\begin{tabular}{lll} 
& $\boldsymbol{P}_{\text {Tr }}$ & $\boldsymbol{M}_{\text {bol }}$ \\
& & \\
क Cen & 0.565 & 0.57 \\
M 15 & 0.565 & 0.57 \\
M 3 & 0.496 & 0.80 \\
M 5 & 0.455 & 0.96 \\
Field Variables & $0.43 ?$ & $1.06 ?$ \\
& $0.40 ?$ & $1.20 ?$ \\
\hline
\end{tabular}

The weak part of this deduction is, however, that I am unable to estimate the systematic errors that may be hidden here though I note the results are all reasonable.

Nevertheless, the best explanation of the differing values of $P_{\mathrm{Tr}}$ in different clusters is that the luminosity is systematically different. It appears that this difference correlates well with the metal content since this is very low for $\omega$ Cen and M 15 and intermediate in $\mathrm{M} 3$ and $\mathrm{M}$ 5. For the field variables, the systematics are less satisfactory and $I$ do not know of a clear determination of $P_{\mathrm{Tr}}$. It seems clear that it is fairly short however, and therefore they are no doubt fainter than $M_{b}=1.0$.

In summary, I would first like to emphasize that the RR Lyrae stars should be segregated according to metal content in establishing their Luminosities. Next I believe we must improve our methods of determining $T_{e}$ from observation. Various modifications of the Wesselink method can provide a very useful value for the stellar radius provided the theory of dynamical systems is employed to establish systematic corrections. Finally, I would say that the nonlinear theory of variables has the possibility of providing new ways of establishing the stellar radius and luminosity, but this theory must be tested and perhaps the models modified by comparison with a few known examples before the results can be believed without question. It is likely that more nonlinear features will be identified and computed, so that more systematic examination of observation for these features can be valuable. 


\section{DISCUSSION}

Iben: The evolutionary calculations which you suggest should be done have, as you well know, been done. They give qualitatively the same dependence on metal abundance as do your rough estimates. Specifically, for the shortest period RR Lyrae stars in clusters such as M 15 and M 92, internal structure calculations suggest $M_{v} \approx+0.5 \mathrm{mag}$. On the basis of evolutionary calculations, the variables in clusters such as M 3 and M 5 should be about 0.25 mag. fainter than those in M 92 and $\mathrm{M} 15$.

Christy: Of course I am acquainted with your work, and I am naturally pleased that it substantiates qualitatively the conclusions to which the pulsation calculations have led us.

Hill: You found a discrepancy between the observed and computed masses for $\mathbf{S}$ Nor which might be resolved by increasing the opacity. Did you use opacities by Cox or by Carson and Stibbs?

Christy: I used the Cox-Stewart opacities. No actual values of opacity, in the range of $T, \rho$ relevant for pulsation, have yet been calculated according to the Stibbs-Carson procedure, but I am very anxious to see such results so that they can be used in pulsation calculations. 